\title{
DAL-1 attenuates epithelial to mesenchymal transition and metastasis by suppressing HSPA5 expression in non-small cell lung cancer
}

\author{
XIAOLIU QIU* ${ }^{*}$ XIAOYING GUAN* , WANXIA LIU* and YAJIE ZHANG \\ Department of Pathology, School of Basic Medical Science, Guangzhou Medical University, \\ Xinzao, Panyu, Guangzhou, Guangdong 511436, P.R. China
}

Received March 13, 2017; Accepted August 10, 2017

DOI: $10.3892 /$ or.2017.6000

\begin{abstract}
Metastasis is the primary cause of death in lung cancer patients and EMT (epithelial-mesenchymal transition) promotes metastasis. Previous study revealed that DAL-1 (differentially expressed in adenocarcinoma of the lung) could attenuate EMT and metastasis in non-small cell lung cancer (NSCLC). Further study proved that HSPA5 (heat shock protein 5), which has a promoting effect on EMT, could bind to DAL-1. In this study, the mRNA and protein expression levels of target molecules were detected by RTq-PCR and western blot assays, the migration and invasion abilities were examined by Transwell migration and invasion assay, and the proliferation ability was measured by CCK- 8 assay. We revealed that DAL-1 was downregulated while HSPA5 was upregulated in NSCLC and found the protein of DAL-1 and HSPA5 co-localized in the cytoplasm and nucleus. We demonstrated that DAL-1 can suppress the expression of HSPA5 on mRNA and protein levels, and decrease EMT, migration, invasion and proliferation abilities by downregulating HSPA5. Furthermore, we discovered that DAL-1 plays a role in inhibiting PI3K/Akt/Mdm2 signaling pathway by suppressing HSPA5.
\end{abstract}

Correspondence to: Professor Yajie Zhang, Department of Pathology, School of Basic Medical Science, Guangzhou Medical University, Xinzao, Panyu, Guangzhou, Guangdong 511436, P.R. China

E-mail: yajiezhang@163.com

${ }^{*}$ Contributed equally

Abbreviations: NSCLC, non-small cell lung cancer; EMT, epithelial-mesenchymal transition; DAL-1, differentially expressed in adenocarcinoma of the lung; HSPA5, heat shock protein 5; Bip, heavy chain binding protein; GRP78, glucose regulated protein 78; HSP70, heat shock protein $70 ; \mathrm{ER}$, endoplasmic reticulum

Key words: DAL-1, HSPA5, epithelial-mesenchymal transition, $\mathrm{PI} 3 \mathrm{~K} / \mathrm{Akt} / \mathrm{Mdm} 2 / \mathrm{p} 53$, metastasis, non-small cell lung cancer

\section{Introduction}

Lung cancer is the main cause of cancer-related mortality worldwide and $80-85 \%$ are NSCLC with five-year survival of only $15 \%$ due to delayed diagnosis and ineffective treatment. Metastasis is the primary cause of death in lung cancer patients (1). A large number of studies have shown that EMT is a prerequisite for the invasion and metastasis of epithelial tumor cells (2-6). The downregulation of epithelial cell markers, such as E-cadherin and $\beta$-catenin, and the upregulation of interstitial cell markers, such as $\mathrm{N}$-cadherin and vimentin, are the mainly molecular features of EMT (7). The downregulation or deletion of E-cadherin is a hallmark of the EMT process and a prerequisite for tumor invasion and metastasis $(8,9)$.

DAL-1 was first identified as a gene lacking in NSCLC by using Differential Display PCR (10). The frequent loss of DAL-1 in lung cancer, breast cancer, gastric cancer, renal clear cell carcinoma and epithelial ovarian cancers suggests that it could be a tumor suppressor (11-15). It has been reported that DAL-1 locus on 18p11.3, and the promoter methylation and loss of heterozygosity are responsible for the inactivation of DAL-1 $(13,16)$. We have demonstrated in previous studies that overexpression of DAL-1 can inhibit the migration and invasion of NSCLC cells by attenuating EMT and found that the HSPA5 protein was a DAL-1-related protein which could be directly bound to the DAL-1 protein (17).

HSPA5, also known as immunoglobulin heavy chain binding protein (Bip) or glucose regulated protein 78 (GRP78), belongs to the heat shock protein 70 (HSP70) family (18). As a stress-induced endoplasmic reticulum (ER) chaperone, HSPA5 is overexpressed in many human cancers including NSCLC. The overexpression of HSPA5 is involved in the tumor progression, such as regulating EMT, proliferation, invasion and metastasis $(19,20)$. HSPA5 has been reported to promote EMT by activating the PI3K/Akt signaling pathway in NSCLC cells (21).

Herein, the expression and co-localization of DAL-1 protein and HSPA5 protein was identified, and the effect of DAL-1 on the expression and functions of HSPA5 were investigated in NSCLC cells. We discovered that the expression of DAL-1 was decreased while the expression of HSPA5 was increased 
in NSCLC cells. We revealed that DAL-1 can inhibit EMT, migration, invasion and proliferation by suppressing HSPA5 expression. We also found that DAL-1 has a role in inhibiting $\mathrm{PI} 3 \mathrm{~K} / \mathrm{Akt} / \mathrm{Mdm} 2$ pathway by depending on HSPA5.

\section{Materials and methods}

Cell lines. The cell lines used in this study (16HBE, A549, SPCA-1, HA579, H520, H460 and H1299) were purchased from American Type Culture Collection (ACTT) and incubated in RPMI-1640 medium containing 10\% feral bovine serum (FBS) (Gibco, Carlsbad, CA, USA), at $37^{\circ} \mathrm{C}, 5 \%$ $\mathrm{CO}_{2}-95 \% \mathrm{O}_{2}$ humid incubator.

Lentivirus expression vectors. The lentivirus expression vectors were purchased from GeneChem Co., Ltd. (Shanghai, China). To obtain stable infection, cells seeded in 96-well plates were infected with $2 \mu \mathrm{l} /$ well lentivirus expression vectors $\left(1 \times 10^{8} \mathrm{TU} / \mathrm{ml}\right)$ using $10 \mu \mathrm{l} /$ well polybrene $(5 \mu \mathrm{g} / \mathrm{ml})$ reagent and $90 \mu \mathrm{l} /$ well RPMI-1640. After $10 \mathrm{~h}$ incubation in serum and antibiotic free condition, the medium with free FBS was replaced by the medium containing $10 \%$ FBS. Cells were incubated with puromycin-containing medium $(2 \mu \mathrm{g} / \mathrm{ml})$ or selected by means of limited dilution to select green fluorescent monoclonal cells to select stable cell lines.

Fluorescent staining. The cased slides were washed three times with PBS in a plate and fixed with $4 \%$ paraformaldehyde for $15 \mathrm{~min}$. After immobilization, the dishes were wash with PBS three times. Normal goat serum was added to the slide and closed at room temperature for $30 \mathrm{~min}$. The blocking solution was removed and a sufficient amount of resistance was added and incubated overnight at $4^{\circ} \mathrm{C}$. The next day, tablets were washed 3 times with PBST, and then fluorescent secondary antibody was added. Incubated at room temperature for $1 \mathrm{~h}$ and then washed with PBST 3 times, DAPI was added for $5 \mathrm{~min}$ in the dark. The plates were again washed with PBST 4 times and then sealed with a sealant containing a fluorescent quencher. Finally, images were observed under a laser confocal microscope.

Western blot analysis. Cells were washed with cold phosphate buffered saline (PBS) and lysed in RIPA buffer with protease inhibitor cocktail (CWBIO, Beijing, China) for $20 \mathrm{~min}$. Cell extracts were separated by $10 \%$ SDS-PAGE gel, and transferred to nitrocellulose membrane $(0.45 \mu \mathrm{m}$ HATF, 10TF of filter; Millipore, Carrigtwohill, Ireland). After blocking in $0.5 \%$ milk for $1 \mathrm{~h}$, the blots were incubated with rabbit anti-DAL-1 mAb (Abcam, Cambridge, MA, USA), rabbit anti-HSPA5 $\mathrm{mAb}$ (Abcam), rabbit anti-E-cadherin $\mathrm{mAb}$ (Cell Signaling Technology, Danvers, MA, USA), rabbit anti- $\beta$-catenin $\mathrm{mAb}$ (Cell Signaling Technology), rabbit anti-N-cadherin mAb (Cell Signaling Technology), rabbit anti-vimentin $\mathrm{mAb}$ (Cell Signaling Technology), rabbit anti-PI3K mAb (Cell Signaling Technology, rabbit anti-p-PI3K mAb (Cell Signaling Technology), rabbit anti-Akt mAb (Cell Signaling Technology), rabbit anti-p-Akt (Ser473) mAb (Cell Signaling Technology), rabbit anti-Mdm2 mAb (Abcam), rabbit anti-p-Mdm2 mAb (Abcam), rabbit anti-p53 mAb (Abcam), rabbit anti- $\beta$-actin $\mathrm{mAb}$ (Cell Signaling Technology). After being washed three
Table I. Sequences of the primers for quantitative reverse transcriptase real-time polymerase chain reaction.

\begin{tabular}{ll}
\hline Genes & \multicolumn{1}{c}{ Sequences } \\
\hline GAPDH & F: CGGAGTCAACGGATTTGGTCGTAT \\
& R: AGCCTTCTCCATGGTGGTGAAGAC \\
DAL-1 & F: TGGCCCAAGGTTCTAAAGATTTCA \\
& R: CAGCTTAAACCCAATGGTGCTTTC \\
HSPA5 & F: GGAATTCGATATGATG \\
& R: GGTAGCTATCA \\
E-cadherin & F: TCCACCACTAGCCAGTATGATGA \\
& R: CACAGTCACACACGCTGACCTCTA \\
B-catenin & F: GGCAGTGCGTTTAGCTGGT \\
& R: TCCACCACTAGCCAGTATGATGA \\
N-cadherin & F: TTGGTTTGGGGAGGGAGA \\
& R: CTGGGGTCAGAGGTGTATCATTT \\
Vimentin & F: AAGACGGTTGAAACTAGAGATGGAC \\
& R: TGCTGGTAATATATTGCTGCACTGA \\
\hline
\end{tabular}

F, forward; R, reverse.

times, blots were further incubated with 1/1000 anti-rabbit IgG (Cell Signaling Technology). Chemiluminescence was measured in a Fusion Solo 2M instrument (Fusion Solo; Vilber Lourmat, Paris, France).

Quantitative real-time reverse transcriptase PCR (RT- $q P C R)$. Total RNA was purified using TRIzol reagent (Takara, Shiga, Japan) according to the manufacturer's instructions. Quantification was performed with a NanoDrop 2000 (Thermo Fisher Scientific, Waltham, MA, USA). RNA (500 ng) was reverse-transcribed using Prime Script TR Reagent kit (RR037A; Takara). The product was used in subsequent RT-qPCR using three duplicate PCR reactions containing $1 \mathrm{X}$ SYBR-Green Master Mix (SYBR Premix Ex Taq II; RR820A; Takara) with the primers in Table I. Relative expression levels of target genes were determined by the $\Delta \Delta \mathrm{Cq}$ method, using GAPDH gene expression to normalize all samples.

Transwell migration and invasion assay. The Transwell insert was coated with $30 \mu \mathrm{l}(1 \mu \mathrm{g} / \mathrm{ml})$ Matrigel (BD Biosciences, Franklin Lakes, NJ, USA) and $2 \times 10^{5}$ cells in $200 \mu \mathrm{l}$ serumfree media were transferred to the top chamber $(6.5 \mathrm{~mm}$; Corning Inc., Corning, NY, USA). The lower chamber was filled with $700 \mu 1$ medium containing 5\% FBS. The cells in the lower chamber were fixed by methanol and stained with Giemsa after culturing for $24 \mathrm{~h}$. For Transwell migration assays, $2 \times 10^{5}$ cells were plated in the top chamber onto the non-coated membrane. The invasion and migrating cells were counted under a microscope from 5 randomly selected fields (magnification, x200).

Cell proliferation assay. Cells $\left(1 \times 10^{3}\right)$ per well were seeded in triplicate into the 96-well plats. After incubation for $24 \mathrm{~h}$, 
A

Comparison of EPB41L3 across 10 analyses

Under-expression

\begin{tabular}{|c|c|c|c|c|c|c|c|c|c|c|c|}
\hline Median rank & $p$-value & Gene & & & & & & & & & \\
\hline 1039.0 & $8.50 \mathrm{E}-10$ & EPB41L3 & & & & & & & & & \\
\hline & & & 1 & 2 & 3 & 4 & 5 & 6 & 7 & & 10 \\
\hline
\end{tabular}

Comparison of EPB41L3 across 10 analyses Over-expression

\begin{tabular}{|c|c|c|c|c|c|c|c|c|c|c|c|}
\hline Median rank & $p$-value & Gene & & & & & & & & & \\
\hline \multirow[t]{2}{*}{11720.0} & 1.000 & EPB41L3 & & & & & & & & & \\
\hline & & & 1 & 2 & 3 & 4 & 5 & 6 & 7 & 8 & 9 \\
\hline
\end{tabular}

$\begin{array}{llllllll}1 & 5 & 10 & 25 & 25 & 10 & 5 & 1\end{array}$

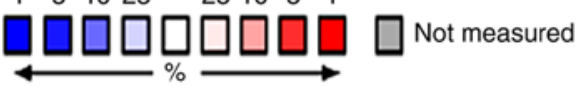

Rank for a gene is the median rank for that gene across each of the analyses.

$\mathrm{p}$-value for a gene is its $\mathrm{p}$-value for the median-ranked analyses.
B

Comparison of HSPA5 across 10 analyses Under-expression

\begin{tabular}{|c|c|c|c|c|c|c|c|c|c|c|c|c|}
\hline Median rank & $\mathrm{p}$-value & Gene & & & & & & & & & & \\
\hline \multirow{2}{*}{7851.5} & 0.559 & HSPAS & & & & & & & & & & \\
\hline & & & 1 & 2 & 3 & 4 & 5 & 6 & 7 & 8 & 9 & 10 \\
\hline
\end{tabular}

Comparison of HSPA5 across 10 analyses Over-expression

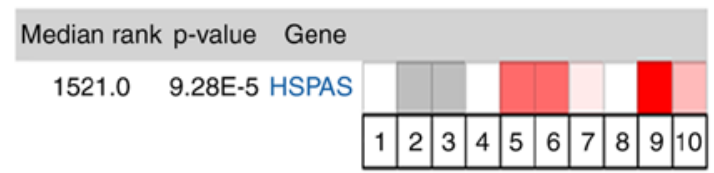

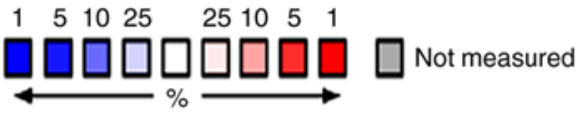

Rank for a gene is the median rank for that gene across each of the analyses.

$p$-value for a gene is its $p$-value for the median-ranked analyses.

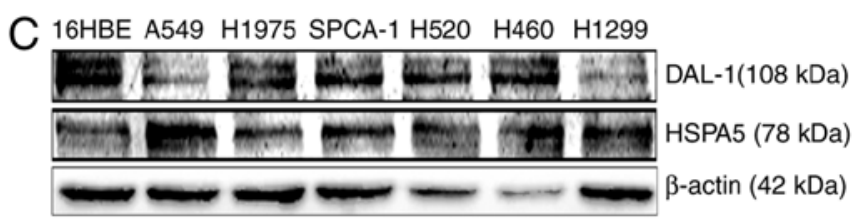

D

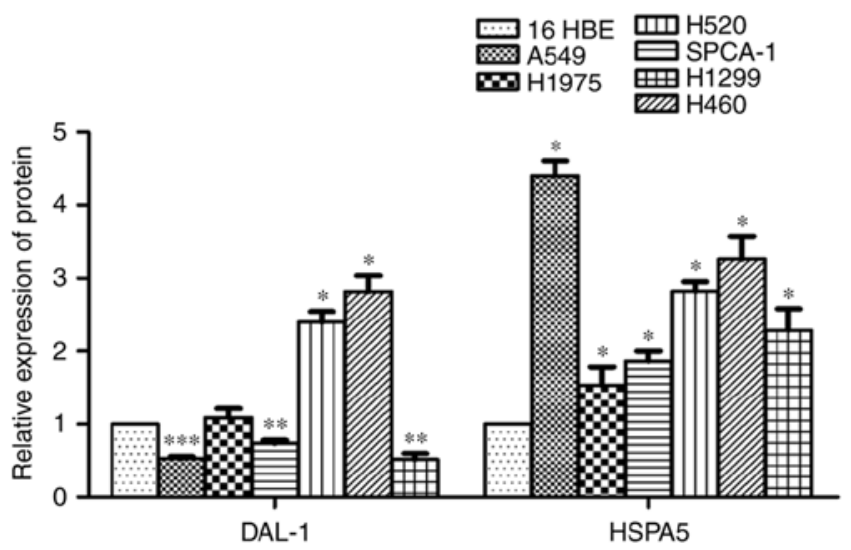

Figure 1. DAL-1 is downregulated while HSPA5 is upregulated in lung cancer. (A) The mRNA expression of DAL-1 in lung cancer tissues compared with the expression in normal tissues. The data came from ten different databases and was analyzed by Oncomine database (http://www.oncomine.org). (B) The mRNA expression of HSPA5 in lung cancer tissues compared with the expression in normal tissues. The data came from ten different databases were analyzed by Oncomine database (http://www.oncomine.org). (C) Western blot analysis of the protein expression of DAL-1 and HSPA5 in NSCLC cells. (D) Statistical analysis of the gray scale of the protein. Data are shown as mean $\pm \mathrm{SD}$ of three independent experiments performed in triplicate. ${ }^{*} \mathrm{P}<0.05,{ }^{* * *} \mathrm{P}<0.005,{ }^{* * * *} \mathrm{P}<0.001$.

the CCK-8 reagent (10 $\mu \mathrm{l} /$ well; CCK-8; Dijindo, Kumamoto, Japan) was added to each well and then incubated for a further $2 \mathrm{~h}$. The quantity of absorbance on the plat was measured at a wavelength of $450 \mathrm{~nm}$ by microplate reader to detect the survival of cells. All experiments were independently repeated at least three times.

Statistical analysis. All analyses were performed by SPSS software (version 13.0; SPSS Inc., Chicago, IL, USA). Data were expressed as mean \pm SD from at least three independent experiments. The difference between different groups was analyzed using a factorial model one-way analysis of variance and P-value $<0.05$ was considered to indicate a statistically significant difference.

\section{Results}

DAL-1 is downregulated while HSPA5 is upregulated in NSCLC. The Oncomine database (http://www.oncomine. org) was utilized to analyze the mRNA expression of DAL-1 and HSPA5 in lung cancer patients. Data from ten databasesdemonstrated that, compared with normal tissues, the mRNA expression of DAL-1 in lung cancer tissues was downregulated $(\mathrm{P}<0.05$; Fig. 1A) while the mRNA expression of HSPA5 was upregulated $(\mathrm{P}<0.05$; Fig. 1B). The western blot assay was used to investigate the protein expression level of DAL-1 and HSPA5 in NSCLC cells (A549, SPCA-1, H1975, H520, H460 and H1299). As shown in Fig. 1C, compared with the immortalized bronchial epithelial cells (16HBE), the protein expression of DAL-1 was lower in A549, SPCA-1 and H1299 cells $(\mathrm{P}<0.05)$ while higher in $\mathrm{H} 520$ and $\mathrm{H} 460$ cells $(\mathrm{P}<0.05)$. The protein expression of HSPA5 was higher in all the NSCLC cells $(\mathrm{P}<0.05)$. Fig. 1D showed a statistical analysis of the gray scale of the protein.

DAL-1 protein and HSPA5 protein are co-localized in the cytoplasm and nucleus of NSCLC cells. Knowing that DAL-1 protein and HSPA 5 protein can be combined directly in NSCLC 

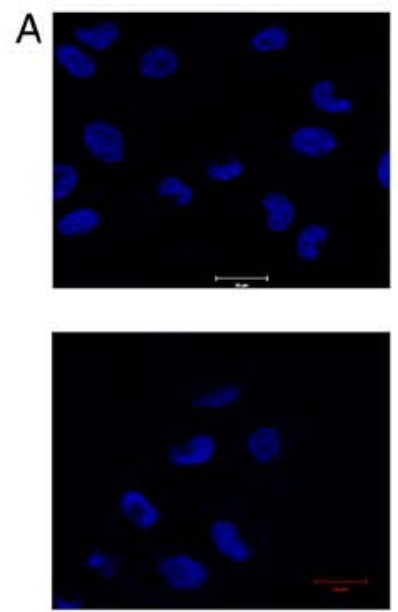

DAPI
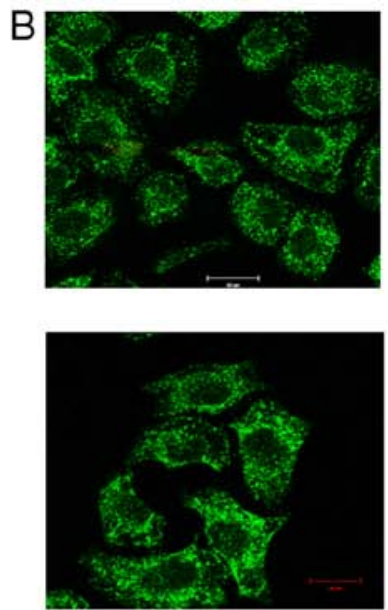

DAL-1
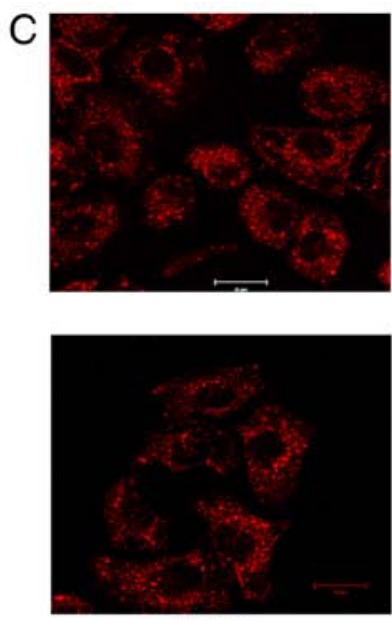

HSPA5
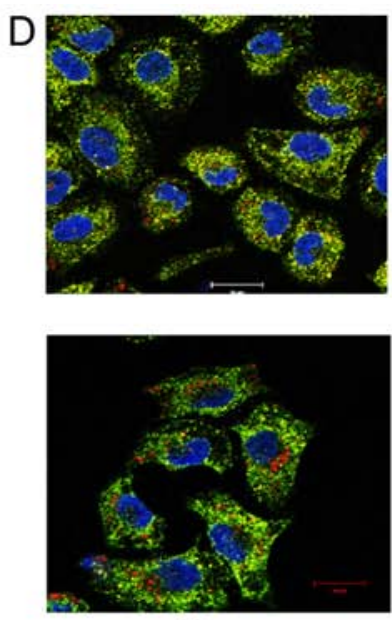

Merge

Figure 2. DAL-1 protein and HSPA5 protein were co-localized in the cytoplasm and nucleus of NSCLC cells. Bar, $20 \mu \mathrm{m}$. (A) DAPI (blue). (B) DAL-1/AlexaFluo-488 (green). (C) HSPA5/Alexa-Fluo-594 (red). (D) Merge (magnification, x100).

cells, we further observed their co-localization in H460 cells which express the two proteins simultaneously. The DAL-1 protein and HSPA5 protein were stained by immunofluorescence and the co-localization of them was observed by laser confocal scanning microscope. The nucleus labeled by DAPI showed blue color (Fig. 2A), DAL-1 proteins were marked by green fluorescence (Fig. 2B) and HSPA5 proteins were labeled by red fluorescence (Fig. 2C). After merging, we can see these two proteins co-localized in cytoplasm and nucleus (Fig. 2D).

Overexpression of DAL-1 suppresses EMT, migration, invasion and proliferation of NSCLC cells. In order to investigate the functional role of DAL-1 in NSCLC cells, a lentivirus vector LV-DAL-1 was established to functionally overexpress DAL-1 in A549 cells. The protein expression of DAL-1 in stably transfected cells (A549/LV-DAL-1) was confirmed by western blot assay (Fig. 3A). The mRNA expression of E-cadherin and $\beta$-catenin were increased while the mRNA expression of $\mathrm{N}$-cadherin and vimentin were decreased in A549/LV-DAL-1 cells compared with control cells (A549/LV-CON1) $(\mathrm{P}<0.05$; Fig. 3C). Similarly, the protein expression of E-cadherin and $\beta$-catenin were upregulated while the protein of $\mathrm{N}$-cadherin and vimentin were downregulated in A549/LV-DAL-1 cells (Fig. 3D). Transwell migration and invasion assays revealed the suppression role of DAL-1 on migration and invasion abilities in A549 cells ( $\mathrm{P}<0.05$; Fig. 3E and F), and CCK-8 cell proliferation assay indicated the suppression role of DAL-1 on proliferation $(\mathrm{P}<0.05$; Fig. 3B). Collectively, these data suggest that DAL-1 plays a role in suppressing EMT, migration, invasion and proliferation in NSCLC cells.

Silence of HSPA5 inhibits EMT, migration, invasion and proliferation ability in NSCLC cells. To investigate the functional role of HSPA5 in NSCLC cells, the lentivirus vectors LV-HSPA5 RNAi-(1/2/3) were established to functionally silence HSPA5 expression in A549 cells. The western blot assay indicated that the protein level of HSPA5 in A549/LV-HSPA5 RNAi-3 decreased most obviously and was used in subsequent experiments (Fig. 4A). The qRT-PCR assay showed that the mRNA expression of E-cadherin and $\beta$-catenin were increased while the mRNA expression of $\mathrm{N}$-cadherin and vimentin were decreased in silenced-HSPA5 cells (A549/LV-HSPA5 RNAi-3) compared with silencedcontrol cells (A549/LV-CON2) ( $\mathrm{P}<0.05 ;$ Fig. 4C). The western blot assay demonstrated that silence of HSPA5 upregulated the protein expression of E-cadherin and $\beta$-catenin, while downregulated the protein expression of $\mathrm{N}$-cadherin and vimentin of A549 cells (Fig. 4D). Transwell migration and invasion assays revealed that HSPA5-silence significantly decreased the migration and invasion abilities of A549 cells $(\mathrm{P}<0.05$; Fig. 4E and F). CCK-8 cell proliferation assay indicated that the proliferation ability was suppressed by silence of HSPA5 $(\mathrm{P}<0.05$; Fig. 4B). These results demonstrate that HSPA5 plays a metastasis and growth promoting role in NSCLC cells.

Overexpression of DAL-1 reduces the expression of HSPA5. To determine whether DAL-1 could influence the expression of HSPA5, we examined the expression of HSPA5 in A549/LV-DAL-1 cells and A549/LV-CON1 cells. The result obtained in the qRT-PCR assay demonstrated that the mRNA expression of HSPA5 was significantly reduced by overexpressing DAL-1 $(\mathrm{P}<0.001$; Fig. $5 \mathrm{~A})$ and western blot assay indicated that the protein expression of HSPA5 was also suppressed by DAL-1 (Fig. 5B). The results suggest that DAL-1 plays a role in suppressing HSPA5 expression.

HSPA5 is involved in DAL-1-mediated EMT, migration, invasion and proliferation inhibition. Knowing that DAL-1 can attenuate while HSPA5 can promote EMT, migration, invasion and proliferation in NSCLC cells, and overexpression of DAL-1 can reduce the mRNA and protein expression of HSPA5, we have reason to believe that DAL-1 can inhibit metastasis and proliferation by suppressing HSPA5 expressing. To elucidate whether HSPA5 is involved in DAL-1-suppressed EMT, migration, invasion and proliferation, A549/LV-DAL-1 cells were stably infected with HSPA5-overexpressed vector LV-HSPA5 (Fig. 6A), and the role of overexpressed HSPA5 in EMT, migration, invasion and proliferation was detected. 

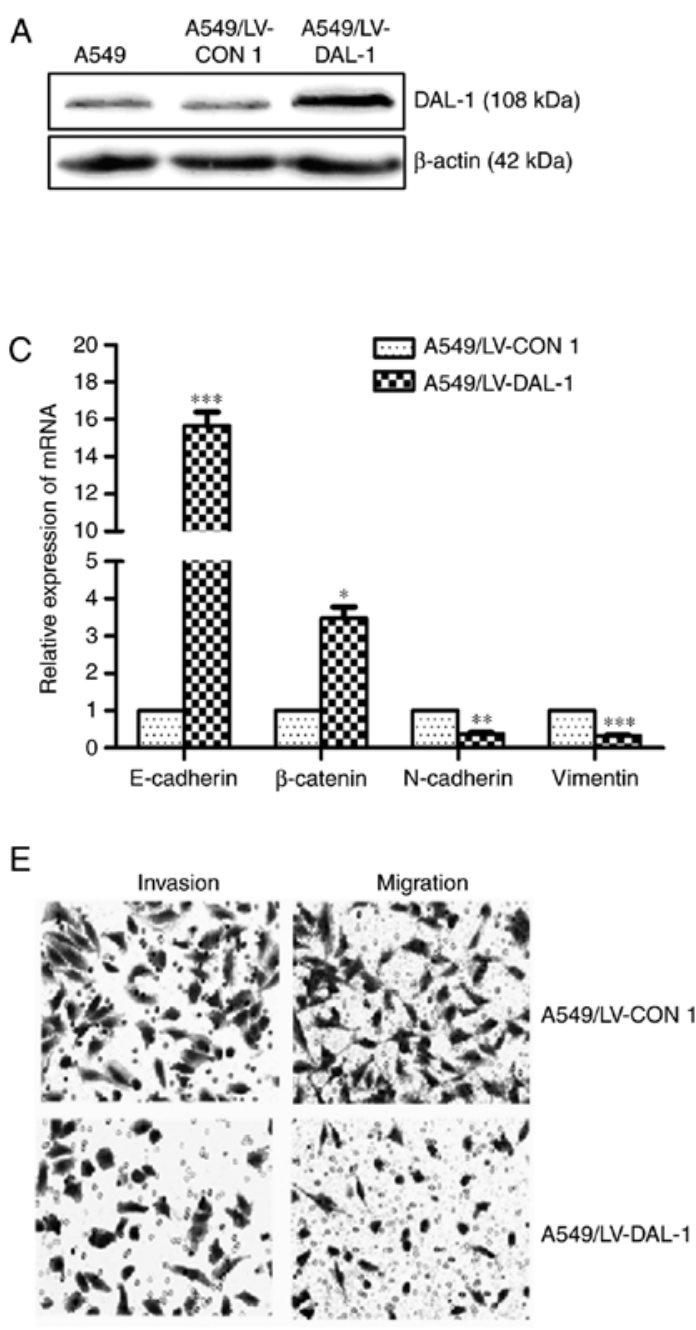
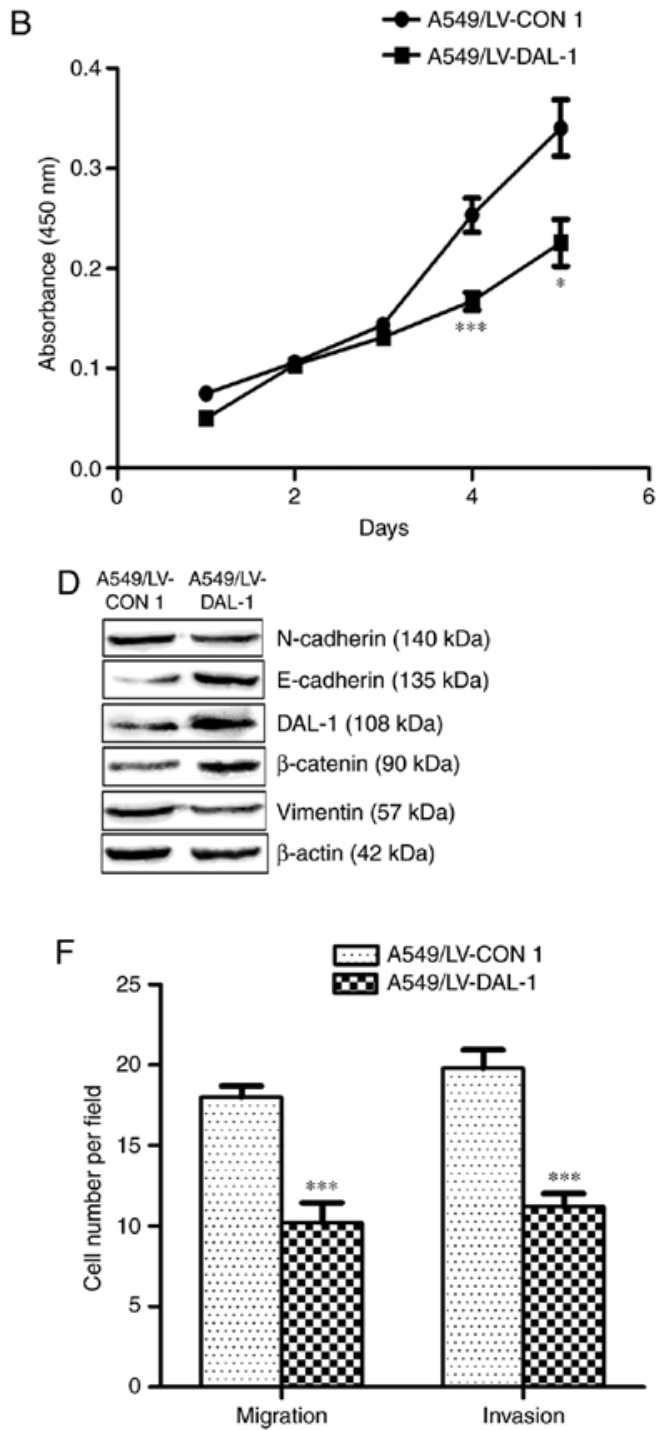

Figure 3. Overexpression of DAL-1 reduces EMT, migration, invasion and proliferation of NSCLC cells. (A) Western blot analysis of DAL-1 protein expression in A549 cells after infecting with LV-DAL-1. (B) The CCK-8 assay was used to measure the effect of DAL-1 on A549 cells proliferation. (C) qRT-PCR was used to measure the effect of DAL-1 on E-cadherin, $\beta$-catenin, N-cadherin and vimentin mRNA expression. (D) Western blot analysis was used to examine the effect of DAL-1 on E-cadherin, $\beta$-catenin, $\mathrm{N}$-cadherin and vimentin protein expression. (E) The Transwell migration and invasion assays were used to measure the effect of DAL-1 on A549 cells migration and invasion (magnification, x200). (F) Statistical analysis of Transwell migration and invasion assays. Data are shown as mean \pm SD of three independent experiments performed in triplicate. ${ }^{*} \mathrm{P}<0.05,{ }^{* *} \mathrm{P}<0.005,{ }^{* * * *} \mathrm{P}<0.001$.

The results of qRT-PCR and western blot assays in Fig. 6C $(\mathrm{P}<0.05)$ and Fig. 6D revealed that the overexpression of HSPA5 downregulated the mRNA and protein expression levels of E-cadherin and $\beta$-catenin while upregulated the mRNA and protein expression levels of $\mathrm{N}$-cadherin and vimentin. The results of Transwell migration and invasion assays indicated that the migration and invasion abilities in A549/LV-DAL-1 cells were obviously increased by overexpressing HSPA5 ( $\mathrm{P}<0.05$; Fig. $6 \mathrm{C}$ and $\mathrm{D})$. The CCK-8 assay indicated the proliferation ability of A549/LV-DAL-1 cells could be promoted by infected LV-HSPA5 (Fig. 6B). These findings support that HSPA5 is a potential target for DAL-1 and involves in DAL-1-mediated EMT, migration, invasion and proliferation in NSCLC cells.

Suppression of HSPA5 is not essential for DAL-1 to inhibit EMT, migration, invasion and proliferation. A549/LV-HSPA5 RNAi-3 cells were infected with the DAL-1 overexpression lentiviral vector LV-DAL-1 (Fig. 7A) to investigate whether the suppression of HSPA5 is necessary for DAL-1 to inhibit the metastasis and growth in NSCLC cells. The results of qRT-PCR and western blot assays shown in Fig. 7C $(\mathrm{P}<0.05)$ and Fig. 7D demonstrated that, after overexpressing DAL-1 in HSPA5-slienced A549 cells, the mRNA and protein expression of E-cadherin and $\beta$-catenin were increased, while the mRNA and protein expression of $\mathrm{N}$-cadherin and vimentin were decreased. Transwell migration and invasion assays shown in Figs. 6E and $7 \mathrm{~F}(\mathrm{P}<0.05)$, and $\mathrm{CCK}-8$ assay shown in Fig. 7B $(\mathrm{P}<0.05)$ revealed that overexpression of DAL-1 decreased the migration, invasion and proliferation ability in A549/LV-HSPA5 RNAi-3 cells. These data indicate that DAL-1 does not depend on HSPA5 to suppress EMT, migration, invasion and proliferation.

Overexpression of DAL-1 and silence of HSPA5 inhibit the PI3K/Akt/Mdm2/p53 signaling pathway. Studies have shown 
A

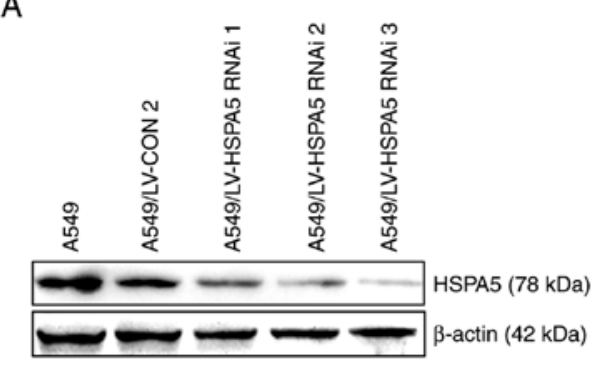

C

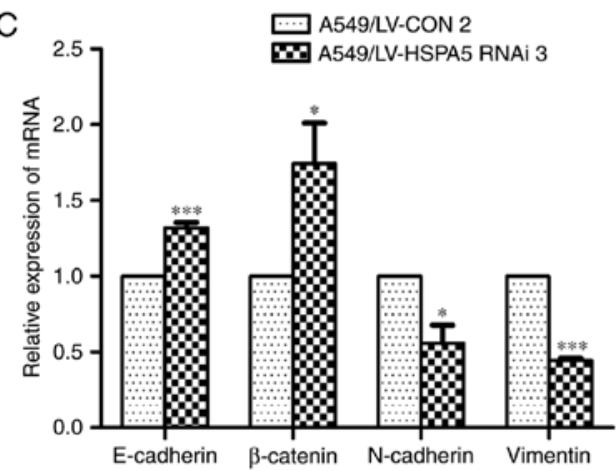

E

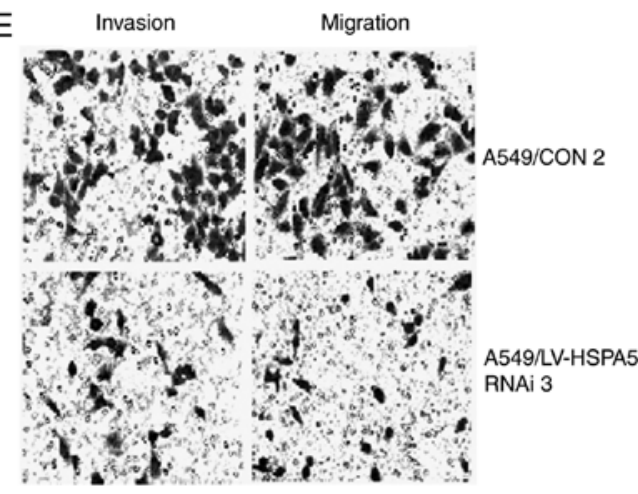

B
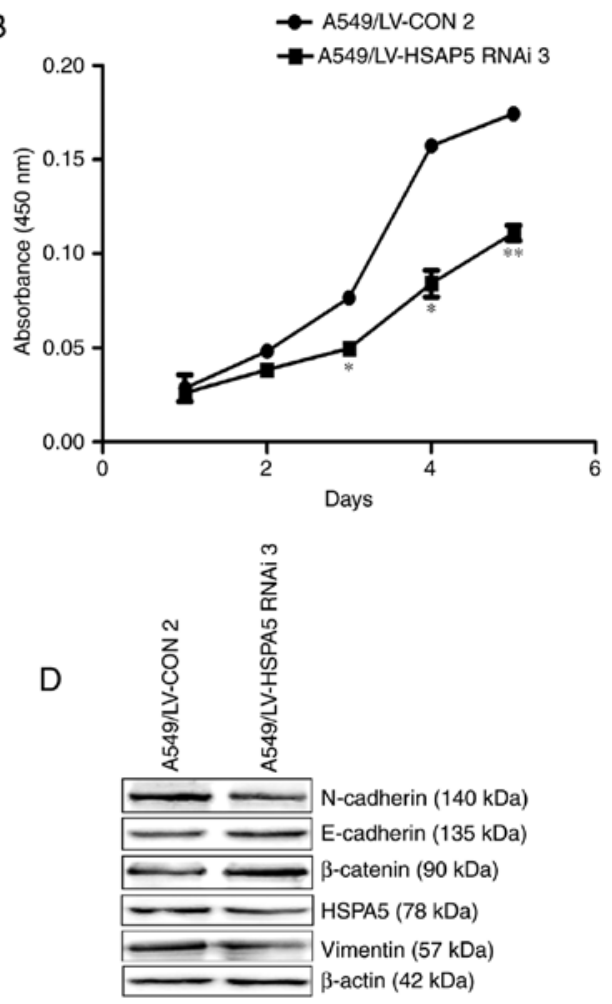

$\mathrm{F}$

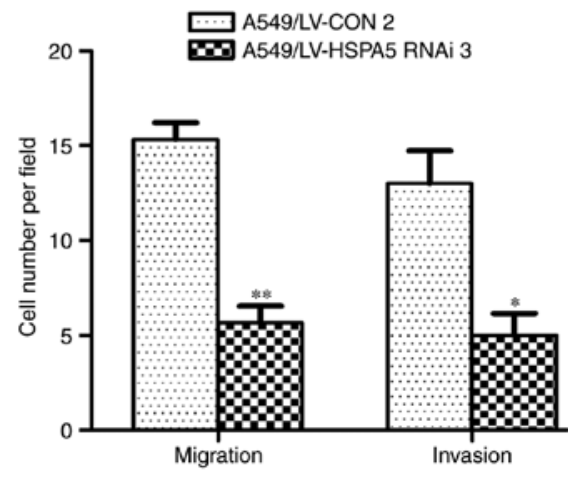

Figure 4. Silence of HSPA5 attenuates EMT, migration, invasion and proliferation in NSCLC cells. (A) Western blot analysis of DAL-1 protein expression in A549 cells after infecting with LV-HSPA5 RNAi-1, LV-HSPA5 RNAi-2 and LV-HSPA5 RNAi-3. (B) The CCK-8 assay was used to measure the effect of silencing-HSPA5 on A549 cells proliferation. (C) qRT-PCR of E-cadherin, $\beta$-catenin, N-cadherin and vimentin. (D) Western blot of E-cadherin, $\beta$-catenin, $\mathrm{N}$-cadherin and vimentin. (E) Transwell migration and invasion assays of the migration and invasion of A549 cells (magnification, $\mathrm{x} 200$ ). (F) Statistical analysis of Transwell migration and invasion assays. Data are shown as mean $\pm \mathrm{SD}$ of three independent experiments performed in triplicate. ${ }^{*} \mathrm{P}<0.05$, ${ }^{* *} \mathrm{P}<0.005,{ }^{* * * *} \mathrm{P}<0.001$.

A

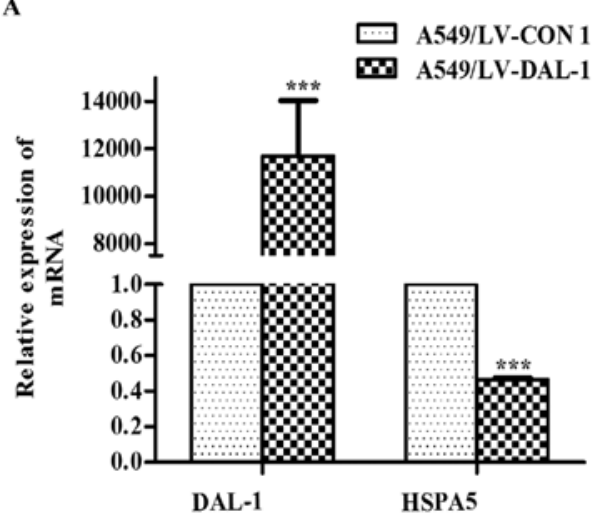

B

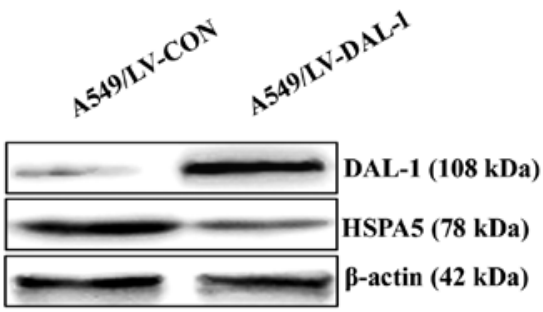

Figure 5. Overexpression of DAL-1 reduces the mRNA and protein expression of HSPA5. (A) qRT-PCR analysis was used to measure the effect of DAL-1 on HSPA5 mRNA and expression. (B) Western blot assay was used to measure the effect of DAL-1 on HSPA5 protein expression. Data are shown as mean \pm SD of three independent experiments performed in triplicate. ${ }^{* * * *} \mathrm{P}<0.001$. 
A

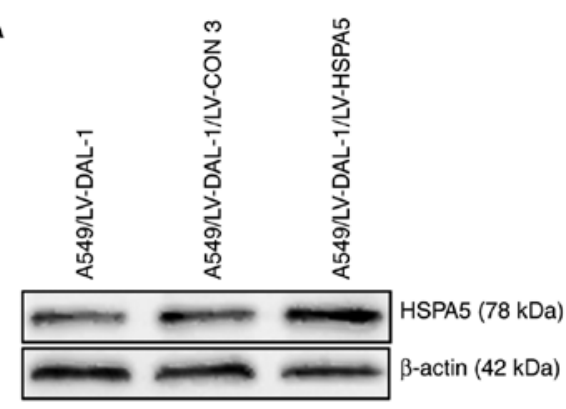

C

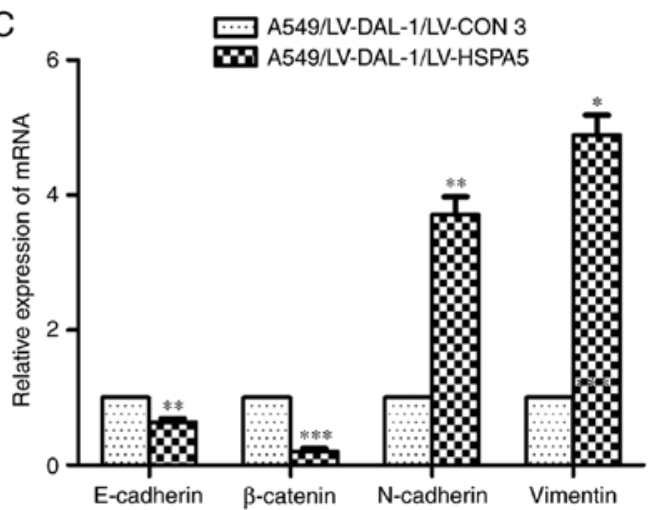

E

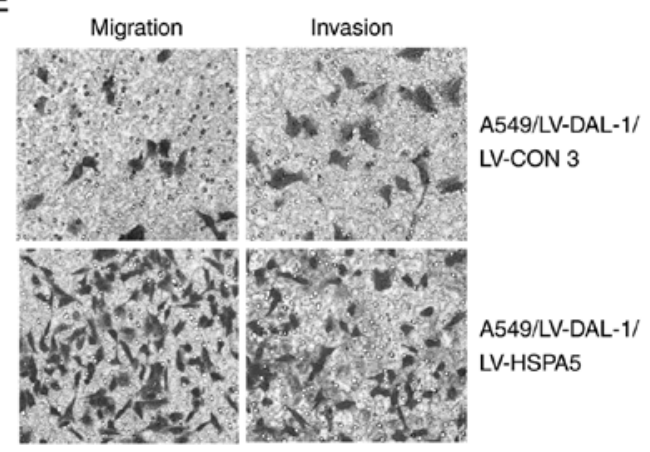

B

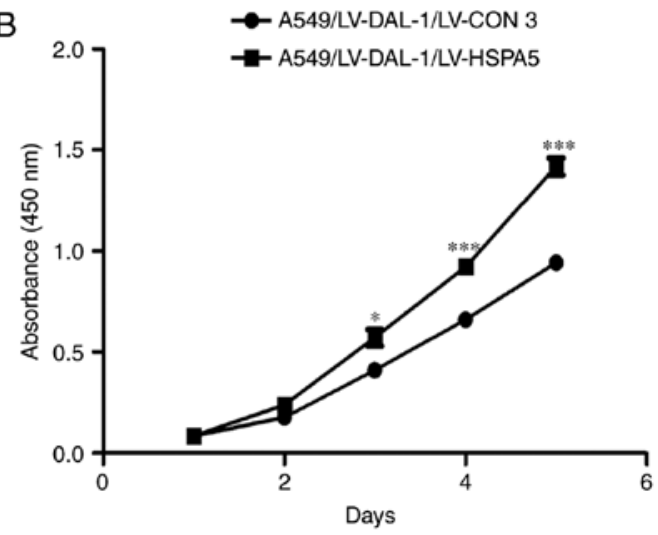

D

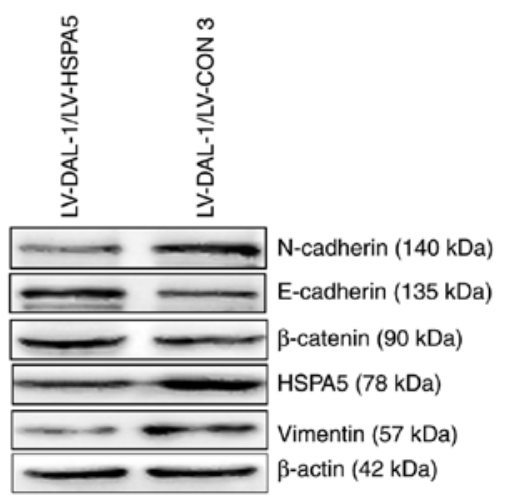

F

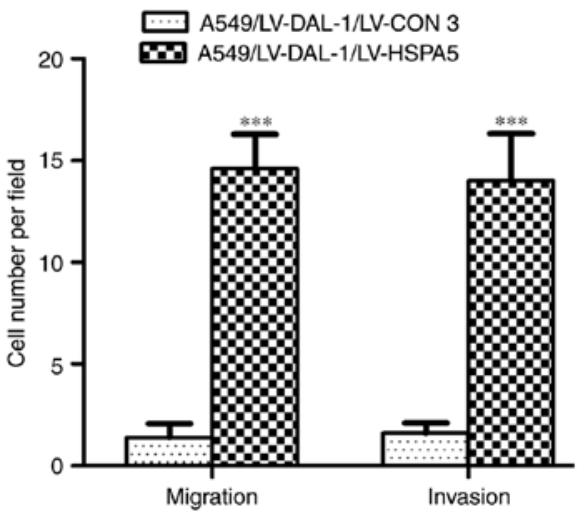

Figure 6. HSPA5 is involved in DAL-1-mediated EMT, migration, invasion and proliferation inhibition. (A) Western blot analysis of HSPA5 protein expression in A549/LV-DAL-1 cells after infecting with LV-HSPA5. (B) The CCK-8 assay of the proliferation of A549/LV-DAL-1/LV-HSPA5 cells and A549/LV-DAL-1/ LV-CON 3 cells. (C) qRT-PCR of the E-cadherin, $\beta$-catenin, N-cadherin and vimentin. (D) Western blot of E-cadherin, $\beta$-catenin, $N$-cadherin and vimentin (E) Transwell migration and invasion assays of the migration and invasion of A549/LV-DAL-1/LV-HSPA5 cells and A549/LV-DAL-1/LV-CON 3 cells (magnification, x200). (F) Statistical analysis of Transwell migration and invasion assays. Data are shown as mean \pm SD of three independent experiments performed in triplicate. ${ }^{*} \mathrm{P}<0.05,{ }^{* *} \mathrm{P}<0.005,{ }^{* * *} \mathrm{P}<0.001$.

that the abnormal activation of PI3K/Akt/Mdm2/p53 signal pathway was closely related to the progression of cancer. It has been reported that HSPA5 can promote lung cancer metastasis by activating PI3K/Akt signaling pathway. However, there is still no report on the effects of DAL-1 on PI3K/Akt/Mdm2/p53 signaling pathway. In this study, western blot assay was utilized to explore the potential roles of DAL-1 and HSPA5 on PI3K/Akt/ Mdm2/p53 signaling pathway in NSCLC cells. The LV-DAL-1 lentivirus vectors transfected into A549 cells displayed an inhibition role on expression of PI3K, p-PI3K, Akt, p-Akt, Mdm2 and $\mathrm{p}-\mathrm{Mdm} 2$ while played a role on upregulating p53 expression (Fig. 8A). Similarly, the lentivirus vectors LV-HSPA5 RNAi3 also functioned reducing the protein expression of PI3K,
p-PI3K, Akt, p-Akt, Mdm2 and p-Mdm2 while increased the expression of p53 protein. These results suggested that DAL-1 could suppress EMT, migration, invasion and proliferation by inhibiting PI3K/Akt/Mdm2/p53 signaling pathway through suppressing HSPA5 expression.

Suppression of HSPA5 is essential for DAL-1 to inhibit PI3K/Akt/Mdm2 signaling pathway. To determine the role of HSPA5 in DAL-1 inhibiting PI3K/Akt/Mdm2/p53 signaling pathway, the effect of LV-HSPA5 on the protein expression of PI3K, p-PI3K, Akt, p-Akt, Mdm2, p-Mdm2 and p53 in A549/LV-DAL-1 cells was investigated. The results shown in Fig. 9A confirmed that overexpressing HSPA5 increased the 
A

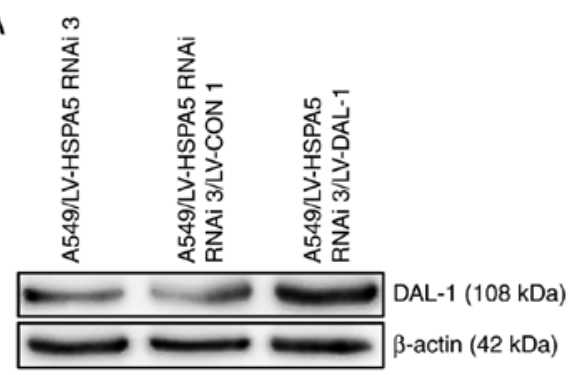

C

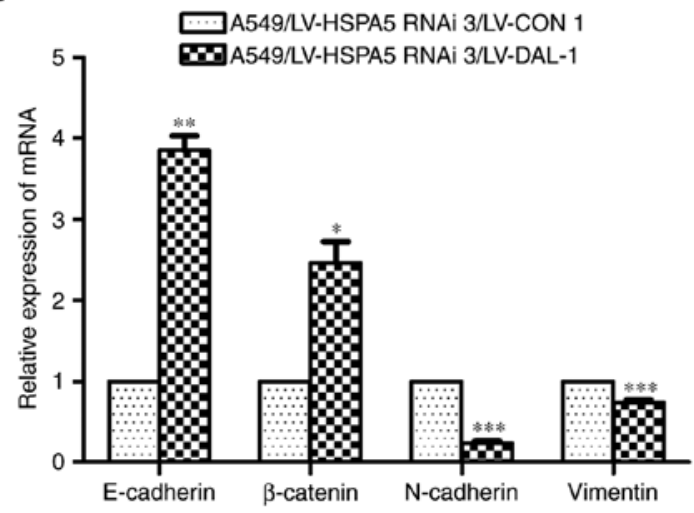

$\mathrm{E}$

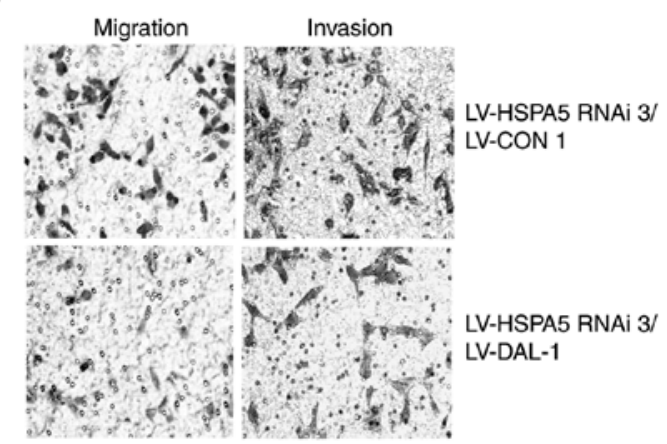

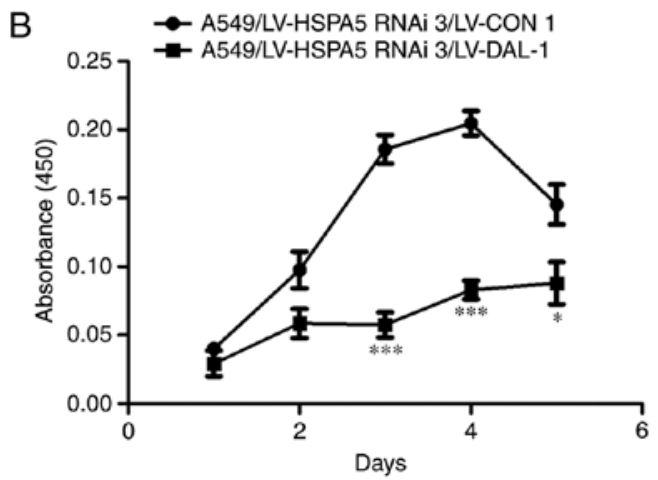

D
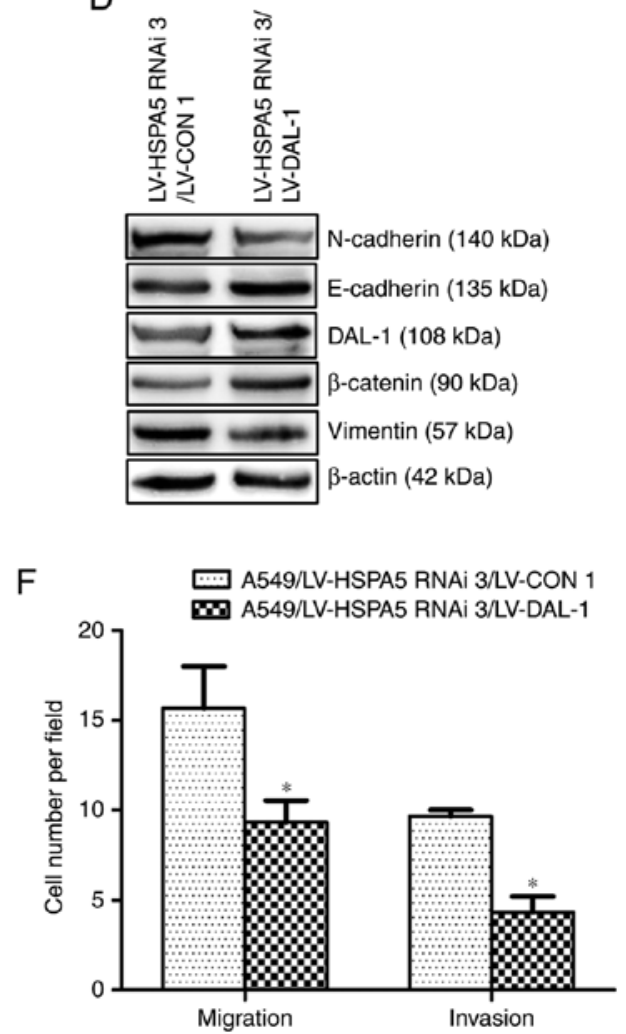

Figure 7. Suppression of HSPA5 is not essential for DAL-1 to suppress EMT, migration, invasion and proliferation. (A) Western blot analysis of DAL-1 protein expression in A549/LV-HSPA5 RNAi-3 cells after infecting with LV-DAL-1. (B) The CCK-8 assay of the proliferation of A549/LV-HSPA5 RNAi-3/LV-DAL-1 cells and A549/LV-HSPA5 RNAi-3/LV-CON 1 cells. (C) qRT-PCR assay of the E-cadherin, $\beta$-catenin, $\mathrm{N}$-cadherin and vimentin. (D) Western blot assay of E-cadherin, $\beta$-catenin, N-cadherin and vimentin. (E) Transwell migration and invasion assays of the migration and invasion abilities of A549/LV-HSPA5 RNAi-3/LV-DAL-1 cells and control cells (magnification, x200). (F) Statistical analysis of Transwell migration and invasion assays. Data are shown as mean $\pm \mathrm{SD}$ of three independent experiments performed in triplicate. ${ }^{*} \mathrm{P}<0.05,{ }^{* * *} \mathrm{P}<0.005,{ }^{* * * *} \mathrm{P}<0.001$.

protein expression of PI3K, p-PI3K, Akt, p-Akt, Mdm2 and $\mathrm{p}-\mathrm{Mdm} 2$ while decreased the protein expression of $\mathrm{p} 53$ in A549/LV-DAL-1 cells, which told us that HSPA5 is involved in DAL-1-inhibited PI3K/Akt/Mdm2/p53 signaling pathway. To further study if HSPA5 is necessary for DAL-1 to regulate the PI3K/Akt/Mdm2 signaling pathway, we examined the protein expression of PI3K, p-PI3K, Akt, p-Akt, Mdm2, p-Mdm2 and p53 in A549/LV-HSPA5 RNAi-3 cells after infected with LV-DAL-1. The results in Fig. 9B show that, after overexpressing DAL-1, the protein expression of PI3K, p-PI3K, Mdm2, p-Mdm2, Akt, p-Akt and p53 in HSPA5silenced cells were all increased, which suggested that the suppression of HSPA5 is necessary for DAL-1 to inhibit PI3K/ $\mathrm{Akt} / \mathrm{Mdm} 2$ signaling pathway.

\section{Discussion}

According to previous studies, the occurrence of EMT is closely related to the metastasis of epithelial malignant tumor. The invasion and metastasis of tumor cells is based on the reduction of cell adhesion and the enhancement of cell motility, and EMT provides conditions for the invasion and metastasis of epithelial tumor cells (2-6). Some studies also reported that EMT can stimulate tumor cells to obtain anti-apoptotic ability and enhance the resistance of tumor cells, but the specific mechanism remains to be further studied $(22,23)$. We have found that DAL-1 can activate the E-cadherin promoter to inhibit EMT, thereby inhibiting the proliferation, invasion and migration abilities in NSCLC cells (17). The aim of this 
A

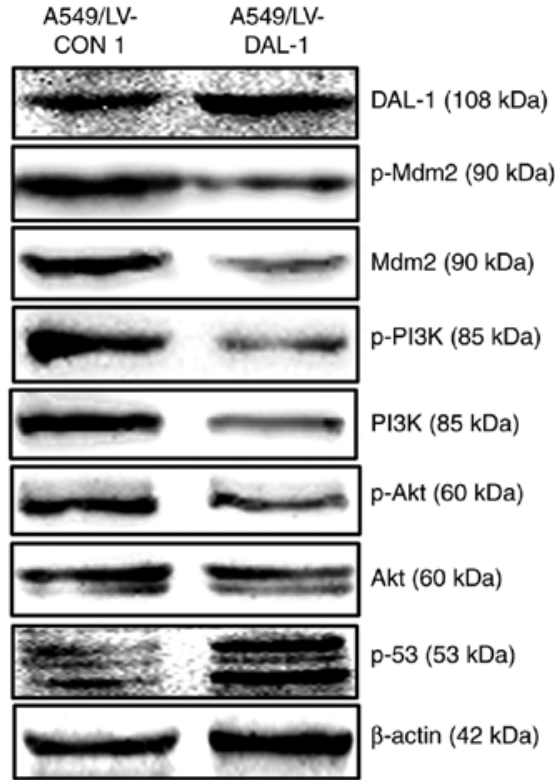

B

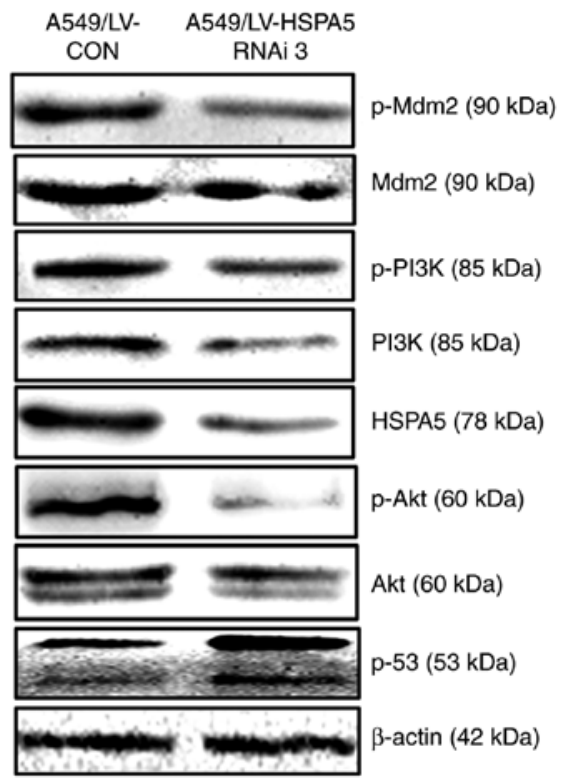

Figure 8. Overexpression of DAL-1 and silence of HSPA5 inhibit the PI3K/Akt/Mdm2/p53 signaling pathway. (A) Western blot assay was used to measure the effect of DAL-1 on the protein expression of PI3K, p-PI3K, Ake, p-Akt, Mdm2, p-Mdm2 and p53 in A549 cells. (B) Western blot assay was used to measure the effect of silencing-HSPA5 on the protein expression of PI3K, p-PI3K, Ake, p-Akt, Mdm2, p-Mdm2 and p53 in A549 cells.
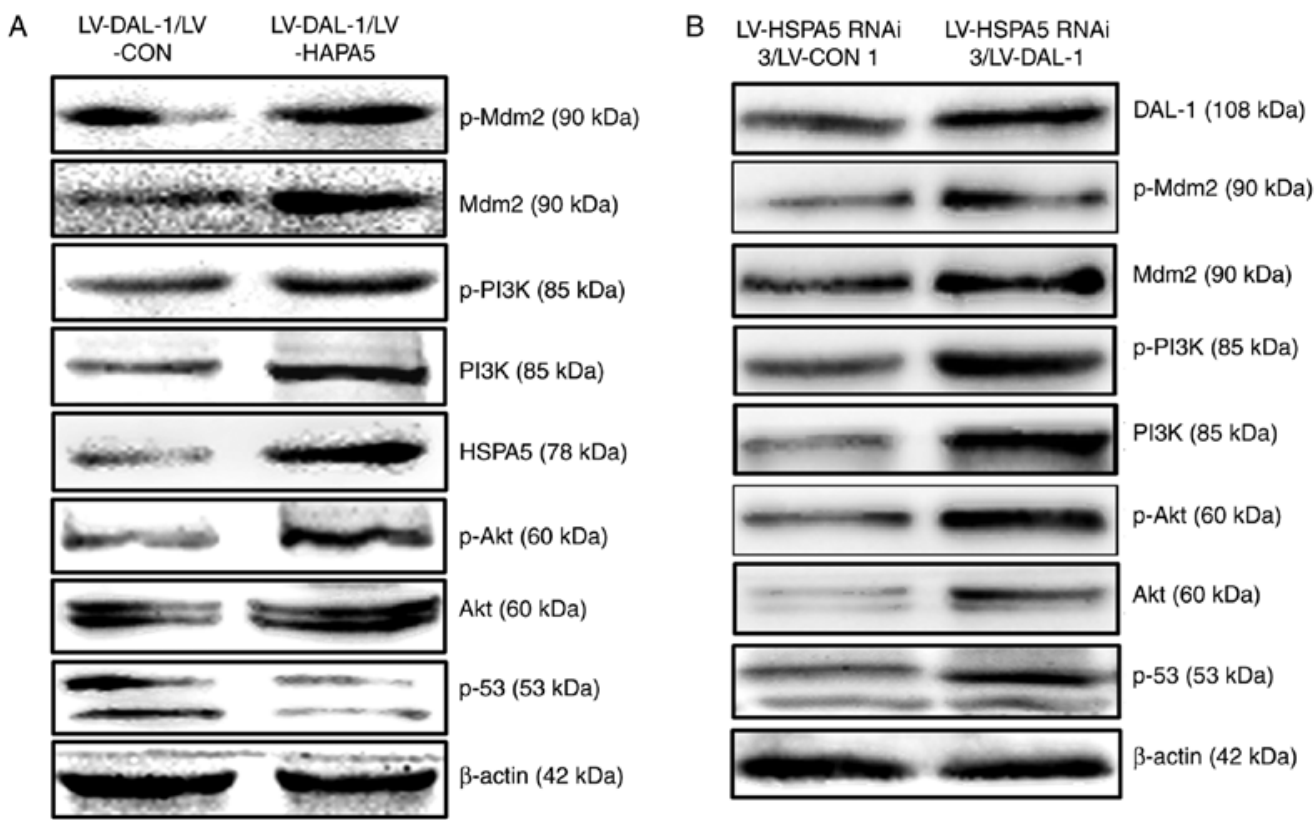

Figure 9. Suppression of HSPA5 is essential for DAL-1 to inhibit PI3K/Akt/Mdm2 signaling pathway. (A) Western blot assay was used to measure the effect of HSPA5 on the protein expression of PI3K, p-PI3K, Ake, p-Akt, Mdm2, p-Mdm2 and p53 in A549/LV-DAL-1 cells. (B) Western blot assay was used to measure the effect of DAL-1 on the protein expression of PI3K, p-PI3K, Ake, p-Akt, Mdm2, p-Mdm2 and p53 in A549/LV-HSPA5 RNAi-3 cells.

study was to further explore the mechanism by which DAL-1 inhibits EMT in NSCLC cells.

In our previous study, HSPA5 was found to be a DAL-1related protein, which can be combined with DAL-1 protein (17). In this study, we investigated the co-localization of DAL-1 protein and HSPA5 protein by laser confocal scanning technique, and found they were co-located in the nucleus and cytoplasm. At present, there is no report on the expression relationship between DAL-1 and HSPA5, and we first studied their expression relationship in NSCLC cells and found that overexpression of DAL-1 can reduce the mRNA and protein of HSPA5. We have proved that DAL-1 can function as a transcription factor to regulate the promoter of E-cadherin (17), which suggested that DAL-1 might inhibit HSPA5 transcription by suppressing the promoter of HSPA5, and the reduction of HSPA5 protein might be caused by the downregulation of mRNA. Double luciferase reporter assay will be used to validate this conjecture.

To investigate the effects of DAL-1 and HSPA5 on EMT, proliferation, invasion and migration in NSCLC cells, we 
overexpressed DAL-1 or silenced the HSPA5 expression of A549 cells, which express a low basal level of DAL-1 but a high basal level of HSPA5. The findings were similar to those of previous studies: DAL-1 inhibited while HSPA5 promoted the EMT, proliferation, invasion and migration of NSCLC cells $(17,24-26)$. We examined the effects of LV-HSPA5 on EMT, migration, invasion and proliferation of DAL-1overexpression cells, and found that overexpression of HSPA5 promoted EMT, migration, invasion and proliferation. We also determined the effects of LV-DAL-1 on EMT, migration, invasion and proliferation of HSPA5-silenced cells, and found that overexpression of DAL-1 decreased EMT, migration, invasion and proliferation. These findings indicate that DAL-1 could inhibit EMT, migration, invasion and proliferation by suppressing HSPA5 expression, but the suppression of HSPA5 was not essential for DAL-1 to inhibit metastasis and growth in NSCLC cells. Studies have shown that DAL-1 can interact with other proteins to play physiological function and anticancer function. For example, DAL-1 protein is reported to be involved in maintaining cell cytoskeleton stability and cell polarity by binding to CADM1, Spectin, actin, Caspr, MPPs (27-30) and mediate cell proliferation, inhibit cell growth by binding to pICIn, CD44 $(31,32)$; by binding to $\beta 8$-integrin, DAL-1 protein can regulate $\beta 8$-integrin localization and the downstream pathway (33).

Emerging evidence indicated that the abnormal activation of PI3K/Akt/Mdm2/p53 signaling pathway is associated with the progression of colon cancer (34). In this study, we first studied the effects of DAL-1 on PI3K/Akt/Mdm2/p53 signaling pathway by western blot assay. The results reveled that overexpression of DAL-1 and silence of HSPA5 decreased the expression of PI3K, p-PI3K, Akt, p-Akt, Mdm2, p-Mdm2 while increased the expression of $\mathrm{p} 53$ protein, which suggested that DAL-1 could attenuate EMT by inhibiting PI3K/Akt/ Mdm2/p53 signaling pathway while HSPA5 could promote EMT by activating PI3K/Akt/Mdm2/p53 signaling pathway in NSCLC cells. We further analyzed the correlation of HSPA5 with the suppression role of DAL-1 in PI3K/Akt/Mdm2/p53 signaling pathway by detecting the effect of LV-HSPA5 on DAL-1-ovexpression cells and the effect of LV-DAL-1 on HSPA5-silenced cells. Our findings point toward an essential role of HSPA5 in DAL-1 inhibiting PI3K/Akt/Mdm2 signaling pathway by suppressing the protein expression levels of PI3K, p-PI3K, Akt, p-Akt, Mdm2 and p-Mdm2.

In this study, DAL-1 was found to suppress the mRNA and protein expression of HSPA5 to inhibit EMT, migration, invasion and proliferation of NSCLC cells. For the first time, the present study revealed the relationship between DAL-1 and $\mathrm{PI} 3 \mathrm{~K} / \mathrm{Akt} / \mathrm{Mdm} 2 / \mathrm{p} 53$ signaling pathway, indicating the potential role of DAL-1 in the inhibition of NSCLC development. Furthermore, it underscores the role of HSPA5 as a potential gene for DAL-1 to inhibit PI3K/Akt/Mdm2 signaling pathway. In conclusion, this study revealed that DAL-1 attenuate EMT, metastasis and proliferation by suppressing HSPA5 expression in NSCLC cells.

\section{Acknowledgements}

This work was funded by the National Nature Science Foundation of China (no. 81401391), the National Nature Science Foundation of Guangdong Province (no. 2015A030313452), China Postdoctoral Science Foundation Grant (no. 2015M570696), and Medical Research Foundation of Guangdong Province (no. B2014188).

\section{References}

1. Al Mohammad B, Brennan PC and Mello-Thoms C: A review of lung cancer screening and the role of computer-aided detection. Clin Radiol 72: 433-442, 2017.

2. Zhu B, Qi L, Liu S, Liu W, Ou Z, Chen M, Liu L, Zu X, Wang J and Li Y: CLASP2 is involved in the EMT and early progression after transurethral resection of the bladder tumor. BMC Cancer 17: 105, 2017.

3. He YX, Song XH, Zhao ZY and Zhao H: HOXA13 upregulation in gastric cancer is associated with enhanced cancer cell invasion and epithelial-to-mesenchymal transition. Eur Rev Med Pharmacol Sci 21: 258-265, 2017.

4. Chen J, Zhang H, Chen Y, Qiao G, Jiang W, Ni P, Liu X and Ma L: miR-598 inhibits metastasis in colorectal cancer by suppressing JAG1/Notch2 pathway stimulating EMT. Exp Cell Res 352: 104-112, 2017.

5. Feng H, Lu JJ, Wang Y, Pei L and Chen X: Osthole inhibited TGF $\beta$-induced epithelial-mesenchymal transition (EMT) by suppressing NF- $\kappa \mathrm{B}$ mediated Snail activation in lung cancer A549 cells. Cell Adhes Migr: Feb 1, 2017 (Epub ahead of print).

6. Xu J, Zhang X, Wang H, Ge S, Gao T, Song L, Wang X, Li H, Qin Y and Zhang Z: HCRP1 downregulation promotes hepatocellular carcinoma cell migration and invasion through the induction of EGFR activation and epithelial-mesenchymal transition. Biomed Pharmacother 88: 421-429, 2017.

7. Yeung KT and Yang J: Epithelial-mesenchymal transition in tumor metastasis. Mol Oncol 11: 28-39, 2017.

8. Otto W, Breyer J, Herdegen S, Eder F, Bertz S, May M, Mayr R, Lausenmeyer EM, Denzinger S, van Rhijn BW, et al: WHO 1973 grade 3 and infiltrative growth pattern proved, aberrant E-cadherin expression tends to be of predictive value for progression in a series of stage T1 high-grade bladder cancer after organ-sparing approach. Int Urol Nephrol 49: 431-437, 2017.

9. Li Z, Yin S, Zhang L, Liu W and Chen B: Prognostic value of reduced E-cadherin expression in breast cancer: A meta-analysis. Oncotarget 8: 16445-16455, 2017.

10. Tran YK, Bögler O, Gorse KM, Wieland I, Green MR and Newsham IF: A novel member of the NF2/ERM/4.1 superfamily with growth suppressing properties in lung cancer. Cancer Res 59: 35-43, 1999.

11. Li L, Li S, Cai T, Wang H, Xie X, Liu Z and Zhang Y: The targeted inhibitory effects of human amniotic fluid stem cells carrying CXCR4 promoter and DAL-1 on non-small cell lung carcinoma growth. Gene Ther 23: 214-222, 2016.

12. Takahashi Y, Iwai M, Kawai T, Arakawa A, Ito T, SakuraiYageta M, Ito A, Goto A, Saito M, Kasumi F, et al: Aberrant expression of tumor suppressors CADM1 and 4.1B in invasive lesions of primary breast cancer. Breast Cancer 19: 242-252, 2012.

13. Wang H, Xu M, Cui X, Liu Y, Zhang Y, Sui Y, Wang D, Peng L, Wang D and Yu J: Aberrant expression of the candidate tumor suppressor gene DAL-1 due to hypermethylation in gastric cancer. Sci Rep 6: 21755, 2016.

14. Nagata M, Sakurai-Yageta M, Yamada D, Goto A, Ito A, Fukuhara H, Kume H, Morikawa T, Fukayama M, Homma Y, et al: Aberrations of a cell adhesion molecule CADM4 in renal clear cell carcinoma. Int J Cancer 130: 1329-1337, 2012.

15. Dafou D, Grun B, Sinclair J, Lawrenson K, Benjamin EC, Hogdall E, Kruger-Kjaer S, Christensen L, Sowter HM, Al-Attar A, et al: Microcell-mediated chromosome transfer identifies EPB41L3 as a functional suppressor of epithelial ovarian cancers. Neoplasia 12: 579-89, 2010.

16. Kittiniyom K, Mastronardi M, Roemer M, Wells WA, Greenberg ER, Titus-Ernstoff L and Newsham IF: Allelespecific loss of heterozygosity at the DAL-1/4.1B (EPB41L3) tumor-suppressor gene locus in the absence of mutation. Genes Chromosomes Cancer 40: 190-203, 2004.

17. Chen X, Guan X, Zhang H, Xie X, Wang H, Long J, Cai T, Li S, Liu Z and Zhang Y: DAL-1 attenuates epithelial-to mesenchymal transition in lung cancer. J Exp Clin Cancer Res 34: 3, 2015. 
18. Moreno JA and Tiffany-Castiglioni E: The chaperone Grp78 in protein folding disorders of the nervous system. Neurochem Res 40: 329-335, 2015

19. Yu T, Guo Z, Fan H, Song J, Liu Y, Gao Z and Wang Q: Cancerassociated fibroblasts promote non-small cell lung cancer cell invasion by upregulation of glucose-regulated protein 78 (GRP78) expression in an integrated bionic microfluidic device. Oncotarget 7: 25593-25603, 2016.

20. Chen HA, Chang YW, Tseng CF, Chiu CF, Hong CC, Wang W, Wang MY, Hsiao M, Ma JT, Chen $\mathrm{CH}$, et al: E1A-mediated inhibition of HSPA5 suppresses cell migration and invasion in triple-negative breast cancer. Ann Surg Oncol 22: 889-898, 2015

21. Liu R, Li X, Gao W, Zhou Y, Wey S, Mitra SK, Krasnoperov V, Dong D, Liu S, Li D, et al: Monoclonal antibody against cell surface GRP78 as a novel agent in suppressing PI3K/AKT signaling, tumor growth, and metastasis. Clin Cancer Res 19: 6802-6811, 2013.

22. Dekervel J, Bulle A, Windmolders P,Lambrechts D, Van Cutsem E, Verslype $\mathrm{C}$ and van Pelt J: Acriflavine inhibits acquired drug resistance by blocking the epithelial-to-mesenchymal transition and the unfolded protein response. Transl Oncol 10: 59-69, 2017.

23. Xu J, Liu D, Niu H, Zhu G, Xu Y, Ye D, Li J, and Zhang Q Resveratrol reverses Doxorubicin resistance by inhibiting epithelial-mesenchymal transition (EMT) through modulating PTEN/Akt signaling pathway in gastric cancer. J Exp Clin Cancer Res 36: 19, 2017.

24. Zhang Y, Xu R, Li G, Xie X, Long J and Wang H: Loss of expression of the differentially expressed in adenocarcinoma of the lung (DAL-1) protein is associated with metastasis of non-small cell lung carcinoma cells. Tumour Biol 33: 1915-1925, 2012.

25. Sun Q, Hua J, Wang Q, Xu W, Zhang J, Zhang J, Kang J and Li M: Expressions of GRP78 and Bax associate with differentiation, metastasis, and apoptosis in non-small cell lung cancer Mol Biol Rep 39: 6753-6761, 2012.

26. Wu HM, Jiang ZF, Fan XY, Wang T, Ke-Xu, Yan XB, Ma Y, Xiao WH and Liu RY: Reversed expression of GRIM-1 and GRP78 in human non-small cell lung cancer. Hum Pathol 45 1936-1943, 2014.
27. Busam RD, Thorsell AG, Flores A, Hammarström M, Persson C, Öbrink B and Hallberg BM: Structural basis of tumor suppressor in lung cancer 1 (TSLC1) binding to differentially expressed in adenocarcinoma of the lung (DAL-1/4.1B). J Biol Chem 286: 4511-4516, 2011

28. Singh V, Miranda TB, Jiang W, Frankel A, Roemer ME, Robb VA, Gutmann DH, Herschman HR, Clarke S and Newsham IF: DAL-1/4.1B tumor suppressor interacts with protein arginine N-methyltransferase 3 (PRMT3) and inhibits its ability to methylate substrates in vitro and in vivo. Oncogene 23: 7761-7771, 2004.

29. Horresh I, Bar V, Kissil JL and Peles E: Organization of myelinated axons by Caspr and Caspr2 requires the cytoskeletal adapter protein 4.1B. J Neurosci 30: 2480-2489, 2010.

30. Kamijo A, Saitoh Y, Ohno N, Ohno S and Terada N: Immunohistochemical study of the membrane skeletal protein, membrane protein palmitoylated 6 (MPP6), in the mouse small intestine. Histochem Cell Biol 145: 81-92, 2016.

31. Jiang W, Roemer ME and Newsham IF: The tumor suppressor DAL-1/4.1B modulates protein arginine N-methyltransferase 5 activity in a substrate-specific manner. Biochem Biophys Res Commun 329: 522-530, 2005.

32. Robb VA, Gerber MA, Hart-Mahon EK and Gutmann DH: Membrane localization of the U2 domain of Protein 4.1B is necessary and sufficient for meningioma growth suppression. Oncogene 24: 1946-1957, 2005.

33. McCarty JH, Cook AA and Hynes RO: An interaction between $\{$ alpha $\}$ v $\{$ beta $\} 8$ integrin and Band $4.1 \mathrm{~B}$ via a highly conserved region of the Band 4.1 C-terminal domain. Proc Natl Acad Sci USA 102: 13479-13483, 2005.

34. Zhang L, Zhu S, Shi X and Sha W: The silence of p66(Shc) in HCT 8 cells inhibits the viability via PI3K/AKT/Mdm-2/p53 signaling pathway. Int J Clin Exp Pathol 8: 9097-9104, 2015. 